\title{
Variación morfométrica intra e interespecífica entre poblaciones de Odontophrynus (Anura: Cycloramphidae) del área central de Argentina
}

\author{
Pablo Raúl Grenat, Nancy Edith Salas \& Adolfo Ludovico Martino \\ Ecología, Departamento de Ciencias naturales, Facultad de Ciencias Exactas, Físico-Químicas y Naturales, \\ Universidad Nacional de Río Cuarto, Ruta Nacional N³6-Km 601, Córdoba, Argentina; pgrenat@exa.unrc.edu.ar, \\ nsalas@exa.unrc.edu.ar, amartino@exa.unrc.edu.ar
}

Recibido 21-X-2011. Corregido 30-III-2012. Aceptado 25-IV-2012.

\begin{abstract}
Intra- and inter-specific morphometric variation between Odontophrynus populations (Anura: Cycloramphidae) of central Argentina. Morphometric analyses are particularly important, and for many years they have supported evolutionary and ecological phenomena, and have been useful for the classification of new species, mainly to the lowest taxonomic levels. In order to assess the degree of sexual dimorphism, the intra-specific morphometric variation and the inter-specific morphological differences, we performed morphometric analyses of two morphologically cryptic species, Odontophrynus cordobae (diploid) and O. americanus (tetraploid). For this, we measured 15 morphometric variables on 211 individuals from 18 localities of Córdoba province. We found sexual dimorphism in six and three parameters in O. cordobae and O. americanus, respectively. Diploid and tetraploid males significantly differed in six morphometric variables. Discriminant Function Analysis (DFA) including all populations correctly classified a $76.37 \%$ of individuals within their respective species. DFA based on four groups (1- allopatric O. cordobae, 2-syntopic O. cordobae; 3- allopatric O. americanus; 4- syntopic $O$. americanus) accurately classified a $73.45 \%$ of individuals within their respective groups. Allopatric individuals of each species and, allopatric and syntopic individuals within each species were clearly segregated. Syntopic individuals of $O$. cordobae and $O$. americanus showed the lowest percentages of correct classification. Morphometric differences between sympatric diploids and tetraploids were not greater than those observed in allopatry. These findings deviate from the expected results under the hypothesis of character displacement, and they suggest that external morphological characters would not have a major influence on the recognition and choice of conspecific males by females. Rev. Biol. Trop. 60 (4): 1589-1601. Epub 2012 December 01 .
\end{abstract}

Key words: Odontophrynus, morphological measures, diploid, tetraploid, cryptic species.

Las especies crípticas, dos o más especies distintas clasificadas erróneamente como una única especie, son aquellas que son extremadamente similares en apariencia (morfología, fisiología y comportamiento) pero se hallan reproductivamente aisladas entre sí (Bickford et al. 2007). Se considera a menudo que las especies crípticas podrían ser el resultado de un proceso reciente de diferenciación (Beheregaray \& Caccone 2007). Sin embargo, la hipótesis de que las especies crípticas son recientes es cuestionada por varios estudios (Bickford et al. 2007), que reportan divergencia aparentemente antigua entre especies crípticas en macabíes
(Colborn et al. 2001), anfípodos (Lefébure et al. 2006) y copépodos (Rocha-Olivares et al. 2001).

El género Odontophrynus, que es endémico de América del Sur, actualmente está compuesto por diez táxones distribuidos al sur y este del continente (Frost 2011), reunidos en tres grupos fenéticos de especies: americanus, cultripes y occidentalis (Savage \& Cei 1965, Cei 1987, Rosset 2008). El grupo americanus está representado por cuatro especies: $O$. americanus, $O$. lavillai, $O$. cordobae y $O$. maisuma. El tetraploide $O$. americanus $(4 n=44)$ de Brasil ha sido el primer caso citado de un poliploide 
natural en especies bisexuales (Sáez \& Brum 1959, Beçak et al. 1966). Posteriormente, Beçak et al. (1970), destacan el hallazgo de una población diploide críptica de $O$. americanus del Brasil. Barrio \& Pistol de Rubel (1972), analizan diferentes poblaciones diplo/tetraploides de $O$. americanus de Argentina y Uruguay, determinando la presencia de la forma diploide en la provincia de Córdoba. La condición tetraploide de $O$. americanus en dicha provincia fue registrada por Barale et al. (1981). Martino \& Sinsch (2002) analizaron poblaciones diploides y tetraploides de $O$. americanus en la zona central de Argentina con el objeto de evaluar si ellas representaban un par de especies crípticas o si todas las poblaciones pertenecían a la misma especie. Dichos autores, al trabajar con variables morfométricas, bioacústicas y moleculares, determinaron que se trataba de dos especies diferentes, nominando la nueva especie (diploide) como O. cordobae.

La especie tetraploide $O$. americanus muestra la mayor distribución geográfica dentro del género encontrándose presentes en los países de Brasil, Paraguay, Uruguay y Argentina (Beçak \& Beçak 1974, Cei 1980, Rosset et al. 2006). En Argentina O. americanus, está presente en varias provincias del centro, este y norte del país (Barrio \& Pistol de Rubel 1972, Lavilla et al. 2000, Martino \& Sinsch 2002, Rosset et al. 2006) y en la provincia de Córdoba es citado por Martino \& Sinsch (2002) hacia el sur de la misma. La distribución de la especie diploide $O$. cordobae está restringida al centro y noroeste de la provincia de Córdoba y un sitio identificado en Villa Ojo de Agua, al sur de Santiago del Estero, Argentina (Martino \& Sinsch 2002, Rosset et al. 2006). El par de especies crípticas $O$. cordobae y $O$. americanus coexisten en sintopía en el centro de Argentina (Grenat et al. 2009b), pero hasta el presente no han sido encontrados híbridos naturales entre estas dos especies.

El tamaño corporal y el canto de advertencia son reconocidos como los principales mecanismos que pueden causar aislamiento pre-reproductivo en anuros (Salas 2004). Varios autores han estudiado el canto de advertencia de O. cordobae y O. americanus (Bogart \& Wasserman 1972, Barrio \& Pistol de Rubel 1972, Martino \& Sinsch 2002), pero los trabajos morfométricos en este complejo críptico de especies son escasos. En este sentido, la morfometría representa un área fundamental de la investigación biológica, particularmente en aquellos estudios relacionados con descripciones cuantitativas, análisis e interpretación de forma o variación de formas (Rohlf 1990). Los datos utilizados en análisis morfométricos son particularmente importantes y durante muchos años han servido como soporte para explicar fenómenos ecológicos y evolutivos y para la clasificación de nuevas especies, principalmente en los niveles taxonómicos más bajos (Lee 1982, Bernal \& Clavijo 2009). La morfometría ha sido útil en la diferenciación de algunas especies morfológicamente crípticas (Laurent 1969, 1975).

Si bien Martino \& Sinsch (2002) evaluaron las diferencias en los caracteres morfológicos entre poblaciones diploides y tetraploides del género Odontophrynus en el área central de Argentina, estos autores analizaron solo dos poblaciones de cada especie. De esta manera los posibles patrones de variación morfológica intraespecífica no fueron estudiados en estas especies ni en otras especies del género. En el presente estudio se evalúa el grado de diferenciación morfológica entre estas dos especies crípticas en base a un mayor número de individuos por especie y considerando poblaciones pertenecientes a 18 sitios de muestreo. Además, se compara la variación morfológica dentro de cada una de las especies, evaluando los posibles patrones de variación geográfica. Debido a que dentro de la muestra analizada se encuentran poblaciones alopátricas y sintópicas de ambas especies, se analizan las diferencias intra e interespecíficas desde la hipótesis del desplazamiento de caracteres reproductivos. En este sentido, de acuerdo a la teoría de desplazamientos de caracteres (Brown \& Wilson 1956), se esperaría una acentuación de las diferencias morfológicas entre poblaciones simpátricas de especies cercanamente relacionadas, debido a una presión selectiva, 
en relación a las diferencias encontradas entre poblaciones alopátricas.

\section{MATERIALES Y MÉTODOS}

Los muestreos para el relevamiento de individuos de Odontophrynus fueron realizados entre los años 2005-2010, durante el período de actividad reproductiva de las especies que ocurre entre los meses de Septiembre y Marzo. Debido a que estas especies presentan hábitos principalmente crepusculares y nocturnos, los muestreos fueron realizados entre el atardecer y la madrugada (8pm-4am). Los sitios de reproducción fueron identificados mediante el reconocimiento del canto de las especies en estudio. Se recorrieron los bordes de los cuerpos de agua y se localizó a los individuos mediante búsqueda activa utilizando linternas frontales (Toledo et al. 2011, Tonini et al. 2011). En cada uno de los sitios se capturaron 8-20 individuos adultos, mediante recolección manual, y se registró las medidas morfométricas in situ. Las mediciones fueron realizadas sobre los organismos vivos, los cuales fueron liberados inmediatamente después de ser medidos (Bosch \& Márquez 1996).

Los individuos pertenecientes a nueve poblaciones de $O$. cordobae fueron obtenidos cubriendo gran parte de su restringida distribución en el centro-norte de la porción occidental de la provincia de Córdoba, Argentina. Los sitios más distantes estuvieron separados aproximadamente por $250 \mathrm{~km}$ en línea recta y la altitud fluctuó desde 450 a $1450 \mathrm{msnm}$. Los sitios de muestreo fueron: Villa General

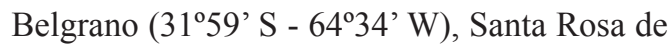

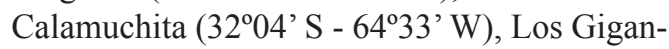
tes $\left(31^{\circ} 23^{\prime} \mathrm{S}-64^{\circ} 21^{\prime} \mathrm{W}\right)$, Tegua (32 $39^{\circ} \mathrm{S}$ $\left.64^{\circ} 18^{\prime} \mathrm{W}\right)$, Berrotarán (3228' S - 64 $\left.23^{\circ} \mathrm{W}\right)$,

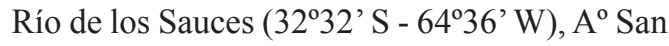
Francisco-Camino Oeste $\left(32^{\circ} 35^{\prime} \mathrm{S}-6^{\circ} 29^{\prime}\right.$ $\mathrm{W})$, Paraje El Cano (32 $\left.37^{\prime} \mathrm{S}-64^{\circ} 35^{\prime} \mathrm{W}\right)$, Paraje La Escondida (32 $\left.40^{\prime} \mathrm{S}-64^{\circ} 31^{\prime} \mathrm{W}\right)$.

Los individuos de $O$. americanus fueron relevados en nueve localidades del Sur de Córdoba y noreste de San Luis, Argentina. Los sitios más distantes estuvieron separados aproximadamente por $145 \mathrm{~km}$ en línea recta y la altitud fluctuó desde 450 a $1350 \mathrm{msnm}$. Los sitios de muestreo fueron: Sampacho $\left(33^{\circ} 23^{\prime}\right.$

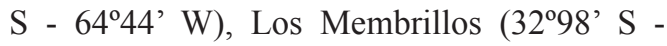
$\left.65^{\circ} 83^{\prime} \mathrm{W}\right)$, Río Cuarto $\left(33^{\circ} 08^{\prime} \mathrm{S}-64^{\circ} 21^{\prime} \mathrm{W}\right)$, Baigorria ( $32^{\circ} 51^{\prime} \mathrm{S}-64^{\circ} 20^{\prime}$ W), Alcira Gigena (324' S - 64 $21^{\circ}$ ' W), Camino A. Corral-La Escondida (32\%40' S - 64\%35' W), Camino

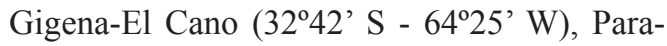
je El Cano $\left(32^{\circ} 37^{\prime} \mathrm{S}-64^{\circ} 35^{\prime} \mathrm{W}\right)$, Paraje La

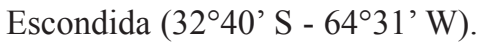

La morfología de las especies de Odontophrynus se analizó con base en 15 variables morfométricas, las cuales fueron obtenidas mediante el uso de un calibrador digital Mahr 16 ES con precisión $0.01 \mathrm{~mm}$ : 1) longitud rostro-cloacal (SVL); 2) ancho máximo de la cabeza (HW); 3) largo de la cabeza (LC); 4) distancia hocico-ojo (HO); 5) distancia internarinas (IN); 6) distancia interocular (IO); 7) distancia ojo-narina $(\mathrm{ON})$; 8) distancia rostronarina (RN); 9) diámetro del ojo (DO); 10) largo del brazo (LB); 11) largo del tercer dedo (TF); 12) largo del fémur (LF); 13) largo de la tibia (LT); 14) largo de la pata (LP) y 15) largo del cuarto dedo del pie (CT) (Martino \& Sinsch 2002). Se probó la normalidad de los datos para cada variable mediante Shapiro-Wilks y se realizaron comparaciones usando ANDEVA para datos con distribución normal o test de Kruskal Wallis para aquellas variables que no presentaron esta distribución. Se calcularon además cinco índices morfométricos en base a los parámetros medidos: 1) relación entre ancho y largo de la cabeza (HW/LC); 2) relación entre las distancias interocular e internarinas ( $\mathrm{IO} / \mathrm{IN}) ; 3$ ) relación entre los miembros anteriores y posteriores $(\mathrm{LB} /(\mathrm{LT}+\mathrm{LF}+\mathrm{LP}))$; 4) relación entre los dedos de mayor longitud en los miembros anterior y posterior $(\mathrm{TF} / \mathrm{CT}) ; 5)$ relación entre el largo de la tibia y del fémur (LT/LF).

Con el objeto de examinar el patrón de variación morfológica dentro de cada especie se realizaron regresiones entre los valores poblacionales promedio de cada variable morfométrica y las coordenadas latitudinales correspondientes a cada sitio. Para la comparación entre poblaciones alopátricas y sintópicas 
de las especies $O$. cordobae y O. americanus, se utilizaron: a) poblaciones alopátricas de la especie $O$. cordobae correspondientes a los sitios de Villa General Belgrano, Santa Rosa de Calamuchita, Los Gigantes y Berrotarán; b) poblaciones alopátricas de la especie $O$. americanus correspondientes a los sitios de Sampacho, Los Membrillos y Río Cuarto y c) poblaciones sintópicas correspondientes a los sitios de El Cano y La Escondida. El análisis discriminante con validación cruzada fue aplicado para obtener una medida adicional de la diferenciación morfológica inter e intraespecífica (Sinsch \& Schneider 1999). Todos los análisis estadísticos se realizaron utilizando los programas STATGRAPHICS ${ }^{\circledR}$ Plus 5.0 y SPSS para Windows, versión 11.5.1.

\section{RESULTADOS}

Las medidas morfométricas promedio obtenidas sobre un total de 211 individuos (Odontophrynus cordobae $n=79 \AA / 14$ 우 $O$. americanus $n=103 \delta^{\lambda} / 15$ ㅇ) pertenecientes a 18 localidades se muestran en el cuadro 1 (por especie y sexo). La especie $O$. cordobae mostró diferencias significativas entre machos y hembras en seis variables morfométricas mientras que $O$. americanus presentó diferencias entre sexo en tres parámetros (Cuadro 1). Las hembras de las dos especies se diferenciaron significativamente en la Distancia Interocular (Cuadro 2).

Debido al bajo número de hembras capturadas y a las diferencias encontradas entre sexos en ambas especies, los posteriores análisis se realizaron sólo teniendo en cuenta a los machos de $O$. cordobae y $O$. americanus. Las variables $\mathrm{DO}(W=0.95, \mathrm{p}=0.00), \mathrm{TF}(W=0.92$, $\mathrm{p}=0.00)$ y $\mathrm{CT}(W=0.95, \mathrm{p}=0.00)$ en $O$. cordobae y ON $(W=0.95, \mathrm{p}=0.01), \mathrm{IN}(W=0.87, \mathrm{p}=0.00)$ y $\mathrm{RN}(W=0.96, \mathrm{p}=0.01)$ en $O$. americanus no mostraron una distribución normal. De las 15 variables morfométricas comparadas mediante ANDEVA o Kruskal-Wallis, seis mostraron diferencias significativas entre los machos de $O$. cordobae y $O$. americanus cuando los individuos de todas la poblaciones fueron incluidos en el análisis (Cuadro 3). Estos parámetros

CUADRO 1

Valores promedio de las variables morfométricas por sexo y ANDEVA para $O$. cordobae $(79 \overbrace{}^{\lambda} / 149)$ y $O$. americanus $(103 \hat{\jmath} / 15$ \% $)$

TABLE 1

Mean values of morphometric variables by sex and ANOVAs for $O$. cordobae $(79 \hat{\jmath} / 14+$ ) and $O$. americanus $(103 \hat{\delta} / 15$ )

\begin{tabular}{lcccccc}
\multicolumn{1}{c}{ Variables } & \multicolumn{3}{c}{ O. cordobae } & \multicolumn{3}{c}{ O. americanus } \\
& Machos & Hembras & $p$ & Machos & Hembras & $p$ \\
Largo hocico-cloaca (SVL) & 46.48 & 48.54 & 0.07 & 45.57 & 46.58 & 0.34 \\
Ancho de la cabeza (HW) & 19.32 & 21.03 & 0.003 & 19.21 & 20.22 & 0.009 \\
Largo de la cabeza (LC) & 14.85 & 15.55 & 0.06 & 15.68 & 15.74 & 0.90 \\
Dist. hocico-ojo (HO) & 7.27 & 7.53 & 0.16 & 7.52 & 7.50 & 0.90 \\
Dist. internarinas (IN) & 2.93 & 3.31 & 0.010 & 3.01 & 3.26 & 0.13 \\
Dist. interocular (IO) & 3.65 & 3.60 & 0.72 & 3.96 & 3.99 & 0.89 \\
Dist. ojo-narina (ON) & 3.18 & 3.67 & 0.0004 & 3.30 & 3.47 & 0.14 \\
Dist. rostro-narina (RN) & 4.32 & 4.51 & 0.24 & 4.32 & 4.72 & 0.018 \\
Diámetro del ojo (DO) & 6.18 & 6.41 & 0.21 & 6.41 & 6.34 & 0.67 \\
Largo del brazo (LB) & 19.87 & 21.88 & 0.0003 & 20.12 & 20.85 & 0.09 \\
Largo del 3 er dedo (TF) & 6.29 & 6.60 & 0.19 & 6.37 & 6.19 & 0.3 \\
Largo del fémur (LF) & 16.79 & 17.56 & 0.09 & 16.45 & 16.99 & 0.21 \\
Largo de la tibia (LT) & 15.14 & 16.11 & 0.003 & 15.40 & 15.50 & 0.71 \\
Largo de la pata (LP) & 24.38 & 26.28 & 0.006 & 24.22 & 25.40 & 0.049 \\
Largo del 4to dedo del pie (CT) & 8.89 & 9.22 & 0.31 & 9.03 & 9.29 & 0.27 \\
\hline
\end{tabular}


CUADRO 2

ANDEVA entre hembras de $O$. cordobae $(n=14)$ y $O$. americanus $(n=15)$

TABLE 2

ANOVA among females of $O$. cordobae $(\mathrm{n}=14)$ and $O$. americanus $(\mathrm{n}=15)$

\begin{tabular}{lc}
\multicolumn{1}{c}{ Variables } & $p$ \\
Largo hocico-cloaca (SVL) & 0.30 \\
Ancho de la cabeza (HW) & 0.16 \\
Largo de la cabeza (LC) & 0.77 \\
Dist. hocico-ojo (HO) & 0.89 \\
Dist. internarinas (IN) & 0.79 \\
Dist. interocular (IO) & 0.04 \\
Dist. ojo-narina (ON) & 0.19 \\
Dist. rostro-narina (RN) & 0.37 \\
Diámetro del ojo (DO) & 0.74 \\
Largo del brazo (LB) & 0.14 \\
Largo del 3 ${ }^{\text {er dedo (TF) }}$ & 0.15 \\
Largo del fémur (LF) & 0.36 \\
Largo de la tibia (LT) & 0.15 \\
Largo de la pata (LP) & 0.14 \\
Largo del 4to dedo del pie (CT) & 0.86 \\
\hline
\end{tabular}

están asociados con las mediciones realizadas sobre la región cefálica de los individuos $\mathrm{y}$ en todos los casos se encontraron valores mayores en $O$. americanus. De los cinco índices calculados solamente HW/LC (ANDEVA, $\left.F_{1,180}=16.78, \mathrm{p}<0.001\right)$ y $\mathrm{LT} / \mathrm{LF}$ (ANDEVA, $\left.F_{1,180}=10.47, \mathrm{p}<0.01\right)$ mostraron diferencias significativas entre especies.

Las regresiones realizadas entre cada parámetro morfológico de $O$. cordobae y las coordenadas latitudinales mostraron una leve tendencia clinal en las variables LC $(r=-0.25$, $\left.R^{2}=0.061, \mathrm{p}<0.05\right)$, HO $\left(r=-0.32, R^{2}=0.105\right.$, $\mathrm{p}<0.01)$, IO $\left(r=-0.23, R^{2}=0.055, \mathrm{p}<0.05\right), \mathrm{LB}$ $\left(r=-0.23, R^{2}=0.052, \mathrm{p}<0.05\right)$. Los valores en estas variables aumentaron en dirección norte-sur. En cambio, la variable DO $(r=0.23$, $\left.R^{2}=0.055, \mathrm{p}<0.05\right)$ evidenció un decrecimiento en la misma dirección. Las restantes variables no mostraron relación directa con las coordenadas latitudinales.

CUADRO 3

ANDEVA/Kruskal-Wallis de 15 variables morfométricas entre las especies: $O$. cordobae $\left(\mathrm{n}=79 \bigcirc^{\Uparrow}\right)$ y $O$. americanus $(\mathrm{n}=103$ ふ)

TABLE 3

ANOVA/Kruskal-Wallis of 15 morphometric variables between $O$. cordobae $(n=79 ð)$ and $O$. americanus $(\mathrm{n}=103 \widehat{)})$

\begin{tabular}{|c|c|c|c|c|}
\hline \multirow{2}{*}{ Variable } & \multicolumn{2}{|c|}{ ANDEVA } & \multicolumn{2}{|c|}{ Kruskal-Wallis test } \\
\hline & $\mathrm{F}$ & $\mathrm{p}$ & $\mathrm{H}$ & $\mathrm{p}$ \\
\hline Largo hocico-cloaca (SVL) & 2.80 & 0.09 & - & - \\
\hline Ancho de la cabeza (HW) & 0.19 & 0.66 & - & - \\
\hline Largo de la cabeza (LC) & 14.60 & 0.00 & - & - \\
\hline Dist. hocico-ojo (HO) & 7.62 & 0.01 & - & - \\
\hline Dist. internarinas (IN) & - & - & 4.58 & 0.03 \\
\hline Dist. interocular (IO) & 14.63 & 0.00 & - & - \\
\hline Dist. ojo-narina $(\mathrm{ON})$ & - & - & 4.31 & 0.03 \\
\hline Dist. rostro-narina ( $\mathrm{RN})$ & - & - & 0.079 & 0.78 \\
\hline Diámetro del ojo (DO) & - & - & 7.18 & 0.007 \\
\hline Largo del brazo (LB) & 1.02 & 0.31 & - & - \\
\hline Largo del $3^{\text {er }}$ dedo (TF) & - & - & 1.81 & 0.17 \\
\hline Largo del Fémur (LF) & 2.05 & 0.15 & - & - \\
\hline Largo de la tibia (LT) & 2.73 & 0.10 & - & - \\
\hline Largo de la pata (LP) & 0.17 & 0.68 & - & - \\
\hline Largo del $4^{\text {to }}$ dedo del pie (CT) & - & - & 1.90 & 0.16 \\
\hline
\end{tabular}


En la especie $O$. americanus, los parámetros IN $\left(r=-0.24, R^{2}=0.058, \mathrm{p}<0.05\right)$ e IO $(r=-$ $\left.0.21, R^{2}=0.046, \mathrm{p}<0.05\right)$ mostraron un ligero aumento clinal de norte a sur, mientras que las restantes variables no mostraron estar correlacionadas con la latitud.

Los individuos sintópicos mostraron diferencias significativas entre especies solamente en la variable longitud rostro-cloacal (SVL), mientras que los individuos alopátricos mostraron diferencias entre especies en las variables largo de la cabeza (LC), distancia internarinas (IN), distancia interocular (IO) y Largo del $4^{\text {to }}$ dedo de la pata (Cuadro 4).

En la comparación dentro de cada especie se encontraron diferencias significativas en seis variables morfométricas entre individuos sintópicos y alopátricos dentro de $O$. americanus, mientras que dentro de $O$. cordobae solo dos variables mostraron diferencias estadísticas. Ambas especies mostraron diferencias en sintopía y alopatría en el ancho de la cabeza y la distancia hocico-ojo (Cuadro 5).

El análisis discriminante incluyendo los datos morfométricos de los individuos (machos) de todas las poblaciones obtenidas para $O$. cordobae y $O$. americanus, mostró una clasificación positiva de los individuos dentro de su respectiva especie del $76.37 \%$. El análisis de validación cruzada muestra una clasificación del $70.3 \%$ de los individuos dentro de sus respectivos grupos. La función discriminante obtenida fue altamente significativa (Correlación Canónica $=0.57, \lambda$ de Wilks $=0.67$, $\mathrm{p}<0.0001)$. Las variables de mayor importancia en la función discriminante obtenida correspondieron a: longitud rostro-cloacal (SVL), largo de la cabeza, largo del fémur y distancia interocular (Fig. 1). De los 26 individuos de $O$. americanus reclasificados como $O$. cordobae, 13 pertenecen a áreas de sintopía, mientras que de los 17 individuos de $O$. cordobae

\section{CUADRO 4}

ANDEVA/Kruskal-Wallis de 15 variables morfométricas entre las especies en sintopía $(O$. cordobae=15ð;

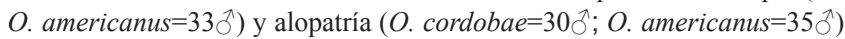

TABLE 4

ANOVA/Kruskal-Wallis of 15 morphometric variables between species in syntopy $\left(O\right.$. cordobae $=15{ }^{\text {; }}$; $O$. americanus $\left.=33 \partial^{\lambda}\right)$ and allopatry $(O$. cordobae $=30 \AA$; $O$. americanus $=35 \overbrace{}^{\lambda})$

\begin{tabular}{|c|c|c|c|c|c|c|c|c|}
\hline \multirow{3}{*}{ Variable } & \multicolumn{4}{|c|}{$\begin{array}{l}\text { O. cordobae vs } O \text {. americanus } \\
\text { (sintopía) }\end{array}$} & \multicolumn{4}{|c|}{$\begin{array}{l}\text { O. cordobae vs } O \text {. americanus } \\
\text { (alopatría) }\end{array}$} \\
\hline & \multicolumn{2}{|c|}{ ANDEVA } & \multicolumn{2}{|c|}{ Kruskal-Wallis } & \multicolumn{2}{|c|}{ ANDEVA } & \multicolumn{2}{|c|}{ Kruskal-Wallis } \\
\hline & $F$ & $p$ & $H$ & $p$ & $F$ & $p$ & $H$ & $p$ \\
\hline Largo hocico-cloaca (SVL) & 4.28 & 0.04 & - & - & 0.55 & 0.46 & - & - \\
\hline Ancho de la cabeza (HW) & 0.04 & 0.85 & - & - & 0.72 & 0.40 & - & - \\
\hline Largo de la cabeza (LC) & 0.87 & 0.35 & - & - & 8.72 & 0.00 & - & - \\
\hline Distancia hocico-ojo (HO) & 0.57 & 0.45 & - & - & 2.27 & 0.14 & - & - \\
\hline Distancia internarinas (IN) & - & - & 0.309 & 0.578 & 7.05 & 0.01 & - & - \\
\hline Distancia interocular (IO) & 0.83 & 0.37 & - & - & - & - & 16.66 & 0.0000 \\
\hline Distancia ojo-narina $(\mathrm{ON})$ & 0.09 & 0.76 & - & - & 2.09 & 0.15 & - & - \\
\hline Distancia rostro-narina $(\mathrm{RN})$ & 2.97 & 0.09 & - & - & 0.53 & 0.47 & - & - \\
\hline Diámetro del ojo (DO) & - & - & 2.29 & 0.13 & 0.43 & 0.51 & - & - \\
\hline Largo del brazo (LB) & 2.35 & 0.13 & - & - & 3.14 & 0.08 & - & - \\
\hline Largo del $3^{\text {er }}$ dedo $(\mathrm{TF})$ & - & - & 1.117 & 0.291 & - & - & 0.59 & 0.44 \\
\hline Largo del fémur (LF) & 0.29 & 0.59 & - & - & 2.43 & 0.12 & - & - \\
\hline Largo de la tibia (LT) & 0.96 & 0.33 & - & - & 0.81 & 0.37 & - & - \\
\hline Largo de la pata (LP) & 2.35 & 0.13 & - & - & 0.62 & 0.43 & - & - \\
\hline$\underline{\text { Largo del } 4^{\text {to }} \text { dedo del pie (CT) }}$ & 3.41 & 0.07 & - & - & - & - & 8.28 & 0.004 \\
\hline
\end{tabular}


CUADRO 5

ANDEVA/Kruskal-Wallis de 15 variables morfométricas entre individuos sintópicos y alopátricos dentro de cada una de las especies

TABLE 5

ANOVA/Kruskal-Wallis of 15 morphometric variables between syntopic and allopatric individuals within each species

\begin{tabular}{|c|c|c|c|c|c|c|c|c|}
\hline \multirow{3}{*}{ Variable } & \multicolumn{4}{|c|}{$\begin{array}{c}\text { O. cordobae } \\
\text { (sintópicos vs alopátricos) }\end{array}$} & \multicolumn{4}{|c|}{$\begin{array}{c}\text { O. americanus } \\
\text { (sintópicos vs alopátricos) }\end{array}$} \\
\hline & \multicolumn{2}{|c|}{ ANDEVA } & \multicolumn{2}{|c|}{ Kruskal-Wallis } & \multicolumn{2}{|c|}{ ANDEVA } & \multicolumn{2}{|c|}{ Kruskal-Wallis } \\
\hline & $\mathrm{F}$ & $\mathrm{p}$ & $\mathrm{H}$ & $p$ & $F$ & $p$ & $H$ & $p$ \\
\hline Largo hocico-cloaca (SVL) & 1.90 & 0.17 & - & - & 0.71 & 0.40 & - & - \\
\hline Ancho de la cabeza (HW) & 4.59 & 0.03 & - & - & 4.25 & 0.04 & - & - \\
\hline Largo de la cabeza (LC) & 0.19 & 0.66 & - & - & 2.53 & 0.11 & - & - \\
\hline Dist. hocico-ojo (HO) & 4.60 & 0.03 & - & - & 5.73 & 0.02 & - & - \\
\hline Dist. internarinas (IN) & 0.02 & 0.90 & - & - & - & - & 7.30 & 0.01 \\
\hline Dist. interocular (IO) & 0.65 & 0.42 & - & - & - & - & 8.09 & 0.00 \\
\hline Dist. ojo-narina (ON) & 0.24 & 0.62 & - & - & 7.40 & 0.01 & - & - \\
\hline Dist. rostro-narina (RN) & 0.15 & 0.70 & - & - & 5.15 & 0.03 & - & - \\
\hline Diámetro del ojo (DO) & - & - & 2.96 & 0.08 & 0.02 & 0.89 & - & - \\
\hline Largo del brazo (LB) & 0.39 & 0.54 & - & - & 0.05 & 0.82 & - & - \\
\hline Largo del $3^{\text {er }}$ dedo $(\mathrm{TF})$ & - & - & 2.76 & 0.09 & 0.00 & 0.96 & - & - \\
\hline Largo del fémur (LF) & 0.67 & 0.42 & - & - & 0.20 & 0.66 & - & - \\
\hline Largo de la tibia (LT) & 0.45 & 0.51 & - & - & 1.25 & 0.27 & - & - \\
\hline Largo de la pata (LP) & 2.89 & 0.10 & - & - & 0.17 & 0.68 & - & - \\
\hline Largo del $4^{\text {to }}$ dedo del pie (CT) & - & - & 0.77 & 0.38 & 0.00 & 0.97 & - & - \\
\hline
\end{tabular}

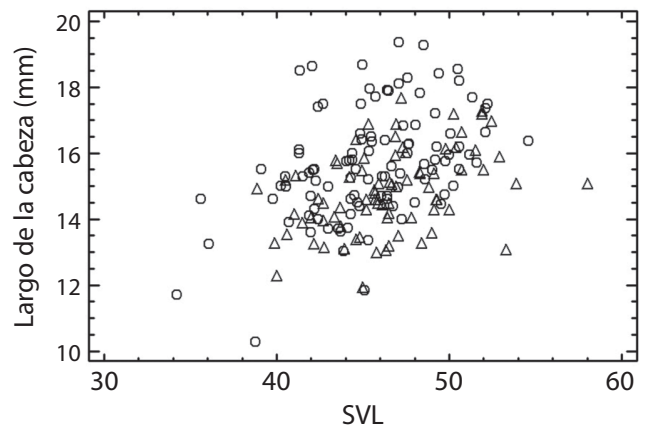

Fig. 1. Segregación morfológica de individuos de las especies $O$. cordobae (triángulos) y $O$. americanus (círculos).

Fig. 1. Morphological segregation of individuals of $O$. cordobae (triangles) and O. americanus (circles).

reclasificados como O. americanus, cuatro corresponden a individuos sintópicos.

A partir del análisis discriminante realizado en base a cuatro grupos (1-O. cordobae alopátrico, 2-O. cordobae sintópico; 3-O. americanus alopátrico; 4-O. americanus sintópico) se obtuvieron tres funciones discriminantes, de las cuales las dos primeras explican un $94 \%$ de la variación (Cuadro 6). La reclasificación de los individuos dentro de cada grupo respectivo fue del 73.45\% (Cuadro 6). Los individuos alopátricos de cada especie se segregan visiblemente, al igual que los individuos alopátricos y sintópicos, dentro de su correspondiente especie. Además, se observa un solapamiento notorio entre los individuos sintópicos de $O$. americanus y $O$. cordobae. Es importante destacar que corresponde a estos grupos un menor porcentaje de clasificación correcta (Cuadro 6, Fig. 2). La Función 1 segregó a los individuos alopátricos de $O$. cordobae de sus coespecificos sintópicos y de todos los individuos de $O$. americanus, mientras que la Función 2 separa a los individuos sintópicos y alopátricos de $O$. americanus. Las variables de mayor aporte fueron, 


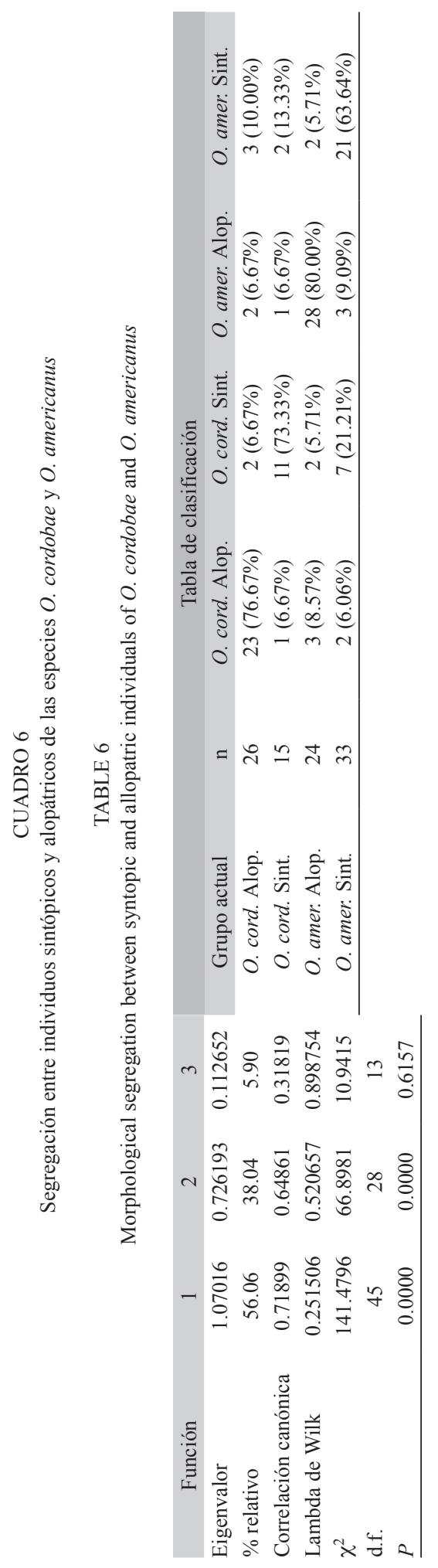

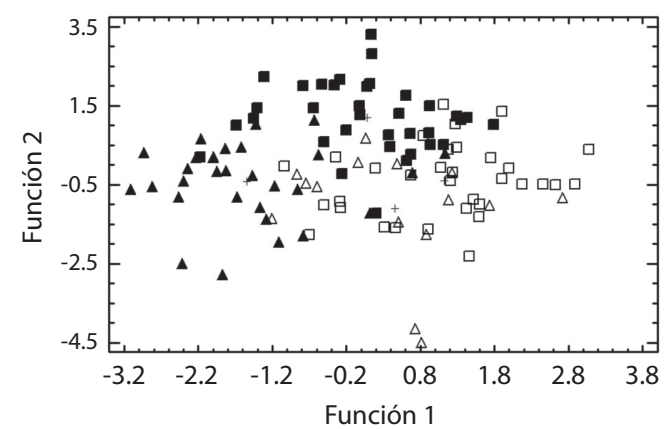

Fig. 2. Segregación morfológica de los individuos alopátricos (símbolos rellenos) y sintópicos (símbolos vacíos) de $O$. cordobae (triángulos) y $O$. americanus (cuadrados). Análisis de Funciones Discriminantes.

Fig. 2. Morphological segregation between allopatric (filled symbols) and syntopic (empty symbols) individuals of $O$. cordobae (triangles) and $O$. americanus (squares). Discriminant Function Analysis.

en la Función 1, el ancho de la cabeza y la distancia hocico-ojo, y en la Función 2, la longitud rostro-cloacal y la distancia interocular. Los resultados obtenidos mediante el análisis de validación cruzada muestran una clasificación del 54\% de los individuos dentro de sus respectivos grupos. Los porcentajes de clasificación correcta de los individuos sintópicos de O. cordobae y $O$. americanus validados $(20 \%$ y $39.4 \%$ respectivamente) fueron notablemente más bajos que los obtenidos en la clasificación original ( $73.3 \%$ y $63.6 \%$ respectivamente). En cambio, los porcentajes de clasificación correcta de los individuos alopátricos de $O$. cordobae y $O$. americanus validados $(76.7 \%$ y $80 \%$ respectivamente) mostraron una menor diferencia con los obtenidos en la clasificación original (73.3\% y $63.6 \%$ respectivamente).

\section{DISCUSIÓN}

Debido a su morfología externa, O. americanus y $O$. cordobae son un típico ejemplo de especies crípticas por su elevado grado de similitud y patrones altamente variables en coloración. Inicialmente, los primeros estudios y comentarios sobre la morfología de especies del género Odontophrynus no reportaron 
diferencias significativas (Gallardo 1966, Beçak et al. 1966, 1970, Barrio \& Pistol de Rubel 1972, Cei 1980, Cei 1985). Martino \& Sinsch (2002), utilizaron mediciones morfométricas como uno de los grupos de caracteres independientes que permitieron diferenciar poblaciones diplo/tetraploides de Odontophry$n u s$, definiendo así la nueva especie $O$. cordobae. Nuestro estudio refuerza el análisis de las diferencias entre las especies $O$. cordobae y $O$. americanus realizado por estos autores e incluye una amplia revisión de especímenes examinados pertenecientes a 18 localidades. Además el amplio ámbito de poblaciones consideradas en este trabajo nos permitió examinar las diferencias morfométricas intraespecíficas dentro de cada especie, un análisis que no había sido abordado hasta este momento. En este sentido, examinar un conjunto de poblaciones a través de la distribución de cada especie, y no solamente poblaciones distantes, permite identificar posibles patrones de variación geográfica que pueden generar confusiones en la interpretación de estudios relacionados con el desplazamiento de caracteres.

Varios autores han realizado estudios comparando la eficiencia de las mediciones morfométricas bajo diferentes condiciones (Lee 1982, Bailey \& Byrnes 1990, Hayek et al. 2001, Schäuble 2004, Bernal \& Clavijo 2009). En estos estudios se reporta que existen diferencias significativas en las mediciones morfométricas dependiendo si son tomadas sobre organismos vivos o preservados, y que las diferencias inter-observador serían mayores que las encontradas en mediciones realizadas por un único observador. En nuestro trabajo, las mediciones fueron realizadas sobre especímenes en condiciones similares y por un único observador.

En la mayoría de las especies de animales dioicos, los machos y las hembras difieren en el tamaño corporal, pero la extensión y la dirección de esta diferencia varía ampliamente dentro y entre especies (Arak 1988). En la mayoría de las especies animales las hembras son más grandes que los machos (Ghiselin 1974). El dimorfismo sexual morfológico es reportado en numerosas especies de anuros en las cuales las hembras (Bosch \& Márquez 1996) o los machos (Taborsky et al. 2009) presentan un mayor tamaño corporal. Martino \& Sinsch (2002), no encontraron diferencias estadísticas entre sexos en $O$. cordobae ni en $O$. americanus, aunque observaron valores morfométricos más altos en las hembras de las dos especies. Estos autores, asumen que esta tendencia observada en los individuos utilizados en su trabajo podría ser efecto del sesgo de la muestra.

En el presente estudio se encontraron diferencias significativas en seis variables morfométricas entre sexos para $O$. cordobae y tres variables para $O$. americanus, resultando mayores los valores de las hembras con respecto al de los machos en todos los casos. En ambas especies, las hembras exhibieron cabezas más anchas que las de los machos. En O. cordobae, las hembras presentaron las extremidades anteriores y posteriores significativamente más largas que las de los machos coespecíficos. Además, la mayoría de las variables que no mostraron diferencias significativas, entre ellas el SVL, también presentaron valores promedio más altos en las hembras de ambas especies. En términos generales, un mayor tamaño por parte de los machos sería esperado si existiera una competencia activa (selección intrasexual) entre machos con encuentros agresivos (Shine 1979, Woolbright 1983, Duellman \& Trueb 1986). En todos los sitios relevados para ambas especies en este estudio no fueron observados encuentros agresivos o competencia física entre machos. Por otra parte, un mayor tamaño de las hembras confiere una ventaja reproductiva en muchos anuros debido a una relación positiva entre el volumen de huevos y el tamaño corporal (Crump 1974).

En el análisis univariado llevado a cabo solamente con los machos de $O$. cordobae y $O$. americanus se encontraron diferencias significativas en las variables morfométricas, relacionadas con la morfología de la cabeza. En estas variables, O. americanus mostró los mayores valores, lo cual podría indicar no solo un mayor tamaño de la cabeza sino también 
una morfología más alargada en un plano longitudinal. Sin embargo no fueron observadas diferencias significativas en el SVL como las obtenidas por Martino \& Sinsch (2002), aunque el valor promedio en esta variable fue levemente mayor en la especie diploide tal como reportaron estos autores. En este sentido, aunque algunos efectos en el tamaño total han sido observados, se describe que muchos poliploides se encuentran dentro de la variación morfológica observada en sus ancestros diploides (Otto \& Whitton 2000). En vertebrados, la poliploidía tiende a tener poco o ningún efecto en el tamaño corporal (Fankhauser 1945; Mahony \& Robinson 1980). Este hecho puede deberse a que, al contrario de lo que ocurre en plantas, en animales poliploides el aumento en el tamaño celular debido al incremento del contenido de DNA suele acompañarse por una disminución en el número total de células manteniendo un tamaño corporal similar al de los progenitores diploides (Fankhauser 1945, Bogart 1980, Mable 2004).

Sinsch et al. (1995), enfatiza la necesidad del uso del análisis multivariado para resolver grupos con similar forma y tamaño. Martino \& Sinsch (2002), usando análisis multivariado reportaron una reclasificación correcta del $75 \%$ de los individuos de $O$. cordobae ( $n$ total $=24$; pertenecientes a las localidades de Villa Gral. Belgrano y Santa Rosa de Calamuchita) y del $81.2 \%$ para individuos de $O$. americanus ( $n$ total $=33$; pertenecientes a las localidades de Río Cuarto y Barreto). Estos autores utilizaron además ejemplares de $O$. occidentalis en el análisis. En el presente estudio, considerando solamente individuos de $O$. cordobae y $O$. americanus pertenecientes a 18 sitios de muestreo, se obtuvo una clasificación positiva del 78.5\% en la especie diploide ( $n$ total $=79$ ) y del $74.8 \%$ en la especie tetraploide ( $n$ total=103). En este sentido, Martino \& Sinsch (2002), consideran que una clasificación inequívoca de los individuos de $O$. cordobae y $O$. americanus no es posible y señalan que la distinción morfológica mejora si la edad del individuo es tomada en cuenta, debido a que los diploides tienden a ser más grandes, en promedio, que los tetraploides de la misma edad.

Diferencias en la morfología de los individuos son reportadas también en el complejo diplo/poliploide Bufo viridis (Castellano et al. 1998, Castellano et al. 2000). Las diferencias encontradas entre las entidades diploides y tetraploides fueron asociadas por los autores a la diferenciación de hábitats evidenciada por las poblaciones de una y otra ploidía. Castellano et al. (1998), sugieren que el mayor tamaño corporal y las patas proporcionalmente más cortas de los diploides respecto a los tetraploides podrían ser el resultado de presiones selectivas que favorecen una reducción de los riesgos de desecación.

En el caso particular de las poblaciones de $O$. cordobae y $O$. americanus consideramos que sus distribuciones no muestran una marcada amplitud y diferenciación de hábitats ni variaciones climáticas significativas. Además, del porcentaje de individuos clasificados incorrectamente, especialmente de $O$. americanus, el $50 \%$ correspondió a ejemplares pertenecientes a zonas de sintopía. Esta diferencia entre individuos tetraploides sintópicos y alopátricos se corresponde con lo obtenido en el análisis univariado.

En el análisis discriminante, considerando grupos sintópicos y alopátricos de cada especie, se obtuvieron porcentajes mayores de clasificación correcta en individuos alopátricos que en individuos sintópicos en ambas especies. Gráficamente se puede observar la diferenciación entre individuos alopátricos de cada una de las especies, y de individuos sintópicos y alopátricos dentro de cada especie. Contrariamente, esta diferenciación no es evidente entre ejemplares sintópicos de $O$. cordobae y $O$. americanus. Esta situación se refleja también en el análisis univariado, en donde se observaron mayores deferencias morfométricas en individuos alopátricos.

Debido a que las diferencias observadas en simpatría entre caracteres morfométricos de diploides y tetraploides no son mayores a las observadas en alopatría, nuestros resultados se alejan de los resultados esperados bajo la 
hipótesis del desplazamiento de caracteres. Adicionalmente, la ausencia de evidencias de combate activo entre machos, reforzado por el menor tamaño de estos en relación a las hembras en ambas especies, podría sugerir que los caracteres morfológicos externos no tendrían una influencia importante en el reconocimiento y elección de machos coespecíficos por parte de las hembras. Bickford et al. (2007), discuten varias razones por las cuales los cambios morfológicos podrían no estar correlacionados con el límite entre especies o bien podrían no ser usados en la discriminación específica. Dos temas parecerían ser generales y recurrentes: las especies crípticas podrían utilizar señales no visuales para la elección de pareja y/o parecerían estar bajo procesos de selección promoviendo la estasis morfológica. En este sentido, a menudo las especies crípticas pueden discriminarse por feromonas o cantos nupciales, y la maquinaria morfológica necesaria para la producción de señales acústicas u olfatorias, no necesariamente difiere de forma apreciable. Las señales visuales son preferiblemente utilizadas por especies de actividad diurna en sitios con poca obstrucción visual (Duellman \& Trueb 1986, Hödl 1996). Las especies $O$. cordobae y $O$. americanus presentan una actividad crepuscular-nocturna, y en muchos casos sus sitios de canto se encuentran protegidos por la vegetación riparia. Además, las diferencias en las señales acústicas encontradas entre estas especies han mostrado ser significativas (Martino \& Sinsch 2002), y podrían estar fuertemente relacionadas con la selección de pareja y el aislamiento reproductivo entre estos taxones crípticos.

\section{AGRADECIMIENTOS}

El primer autor agradece al Consejo Nacional de Investigaciones Científicas y Tecnológicas (CONICET) por la Becas de Posgrado otorgadas. A Julián Valetti por su valiosa ayuda en el campo. La Secretaría de Ciencia y Técnica (SECyT) de la Universidad Nacional de Río Cuarto financia el proyecto en el cual se enmarca el presente estudio (PPI 18/C350).
Este estudio fue autorizado por la Secretaría de Ambiente de la provincia de Córdoba.

\section{RESUMEN}

El análisis morfométrico ha servido como apoyo para explicar fenómenos ecológicos y evolutivos y para la clasificación de nuevas especies. Nosotros realizamos el análisis morfométrico de dos especies morfológicamente crípticas con el objeto de evaluar el grado de diferenciación intra e interespecífica. Se midieron 15 variables sobre 211 individuos pertenecientes a 18 localidades del centro de Argentina. Se encontró dimorfismo sexual en seis variables morfométricas en Odontophrynus cordobae y en tres variables en $O$. americanus. Se encontraron diferencias significativas entre especies en seis variables. El análisis discriminante incluyendo los individuos de todas las poblaciones relevadas para $O$. cordobae y $O$. americanus, mostró una clasificación positiva de los individuos dentro de su respectiva especie del $76.37 \%$. En el análisis discriminante realizado con base en cuatro grupos (O. cordobae alopátrico y sintópico y $O$. americanus alopátrico y sintópico) se obtuvo una reclasificación de los individuos dentro de cada grupo del $73.45 \%$. Las diferencias morfométricas entre especies fueron mayores en sintopía. Los resultados se alejan de los resultados esperados bajo la hipótesis del desplazamiento de caracteres y podrían sugerir que los caracteres morfológicos externos no tendrían una influencia importante en el reconocimiento y elección de machos coespecíficos por parte de las hembras.

Palabras clave: Odontophrynus, medidas morfométricas, diploide, tetraploide, especies crípticas.

\section{REFERENCIAS}

Arak, A. 1988. Sexual dimorphism in body size: A model and a test.Evolution 42: 820-825.

Bailey, R.C. \& J. Byrnes. 1990. A new, old method for assessing measurement error in both univariate and multivariate morphometric studies. Syst. Zool. 39: 124-130.

Barale, G., G.A. Maria, I.E. di Tada \& J.A. Lisanti. 1981. Presencia de Odontophrynus americanus (Anura, Leptodactylidae) tetraploide en la provincia de Córdoba. Rev. UNRC 1: 121-125.

Barrio, A. \& D. Pistol de Rubel. 1972. Encuesta cariotípica de poblaciones argentino-uruguayas de Odontophrynus americanus; (Anura, Leptodactylidae) relacionada con otros rasgos taxonómicos. Physis 31: 281-291.

Beçak, M.L. \& W. Beçak. 1974. Studies of polyploid amphibians-karyotype evolution and phylogeny of the genus Odontophrynus. J. Herpetol. 8: 337-341. 
Beçak, M.L., W. Beçak \& M.N. Rabello. 1966. Cytological evidence of constant tetraploidy in the bisexual South American frog Odontophrynus americanus. Chromosoma 19: 188-193.

Beçak, M.L., W. Beçak \& L.D. Vizotto. 1970. A diploid population of the polyploid amphibian Odontophrynus americanus and the artificial intraespecific triploid hybrid. Experientia 26: 545-546.

Beheregaray, L.B. \& A. Caccone. 2007. Cryptic biodiversity in a changing world. J. Biol. 6: 9.1-9.5.

Bernal, M.H. \& J.A. Clavijo. 2009. An essay on precision in morphometric measurements in anurans: interindividual, intra-individual and temporal comparisons. Zootaxa 2246: 32-44.

Bickford, D., D.J. Lohman, N.S. Sodhi, P.K.L. Ng, R. Meier, K. Winke, K.K. Ingram \& I. Das. 2007. Cryptic species as a window on diversity and conservation. Trends Ecol. Evol. 22: 148-155.

Bogart, J.P. 1980. Evolutionary significance of polyploidy in amphibians and reptiles, p. 341-378. In W.H. Lewis (ed.). Polyploidy, Biological Relevance. Plenum, Nueva York, EEUU.

Bogart, J.P. \& A.O. Wasserman. 1972. Diploid-polyploid species pairs: A possible clue to evolution by polyploidization in anuran amphibians. Cytogenetics 11: 7-24.

Bosch, J. \& R. Márquez. 1996. Discriminant functions for sex identification in two midwife toads (Alytes obstetricans and A. cisternasii). Herpet. J. 6: 105-109.

Brown, W.L. \& E.O. Wilson. 1956. Character displacement. Syst. Zool. 5: 49-64

Castellano, S., C. Giacoma, T. Dujsebayeva, G. Odierna \& E. Balletto. 1998. Morphometrical and acoustical comparison between diploid and tetraploid green toads. Biol. J. Linn. Soc. 63: 257-281.

Castellano, S., C. Giacoma \& T. Dujsebayeva. 2000. Morphometric and advertisement call geographic variation in polyploid green toads. Biol. J. Linn. Soc. 70: $341-360$

Cei, J.M. 1980. Amphibians of Argentina. Monit. Zool. Ital. 2: 1-609.

Cei, J.M. 1985. Un nuevo y peculiar Odontophrynus de la Sierra de Guasayán, Santiago del Estero, Argentina (Anura, Leptodactylidae). Cuad. Herp. 1: 1-13.

Cei, J.M. 1987. Additional notes to Amphibians of Argentina: An update, 1980-1986. Monit. Zool. Ital. 21: 209-272.

Colborn, J., R.E. Crabtree, J.B. Shaklee, E. Pfeiler \& B.W. Bowen. 2001. The evolutionary enigma of bonefishes (Albula spp.): cryptic species and ancient separations in a globally distributed shorefish. Evolution 55: 807-820.
Crump, M.L. 1974. Reproductive strategies in a tropical community. Miscellaneous Publication 61, University of Kansas Museum of Natural History, Lawrence, Kansas, EEUU.

Duellman, W.E. \& L. Trueb 1986. Biology of Amphibians. McGraw Hill, Nueva York, EEUU.

Fankhauser, G. 1945. The effects of changes in chromosome number on amphibian development. Q. Rev. Biol. 20: 20-78.

Frost, D.R. 2011. Amphibian Species of the World: an Online Reference. Version 5.5 American Museum of Natural History, Nueva York, EEUU. (Consultado: October 2, 2011, http://research.amnh.org/vz/ herpetology/amphibia/).

Gallardo, J.M. 1966. Zoogeografía de de los Anfibios Chaqueños. Physis 26: 67-81.

Ghiselin, M.T. 1974. The economy of nature and the evolution of sex. University of California, Berkeley, California, EEUU.

Hayek, L., W.R. Heyer \& C. Gascon. 2001. Frog morphometrics: a cautionary tale. Alytes 18: 153-177.

Laurent, R.F. 1969. Diferencias morfológicas entre especies crípticas de los géneros Pleurodema y Physalaemus. Acta Zool. Lill. 25: 81-96.

Laurent, R.F. 1975. Diferencias biométricas entre dos poblaciones similares de Pleurodema. Acta Zool. Lill. 31: 99-106.

Lavilla, E.O., M.L. Ponssa, D. Baldo, N. Basso, A. Bosso, J. Céspedez, J.C. Chebez, J. Faivovich, L. Ferrari, R. Lajmanovich, J.A. Langone, P. Peltzer, C. Ubeda, M. Vaira \& F. Vera Candioti. 2000. Categorización de los anfibios de Argentina, p. 11-34. In E.O. Lavilla, E. Richard \& G.J. Scrocchi (eds.). Categorización de los anfibios y reptiles de la República Argentina. Asociación Herpetológica Argentina, Tucumán, Argentina.

Lee, J.C. 1982. Accuracy and precision in anuran morphometrics: artifacts of preservation. Syst. Zool. 31: 266-281.

Lefébure, T., C.J. Douady, M. Gouy, P. Trontelj, J. Briolay \& J. Gilbert. 2006. Phylogeography of a subterranean amphipod reveals cryptic diversity and dynamic evolution in extreme environments. Mol. Ecol. 15: 1797-1806.

Mable, B.K. 2004. Why polyploidy is rarer in animals than in plants: myths and mechanisms. Biol. J. Linn. Soc. 82: 453-466.

Mahony, M.J. \& E.S. Robinson. 1980. Polyploidy in the Australian leptodactylid frog genus Neobatrachus. Chromosoma 81: 199-212.

Martino, A.L. \& U. Sinsch. 2002. Speciation by polyploidy in Odontophrynus americanus. J. Zool. 257: 67-81.

Otto, S. \& J. Whitton. 2000. Polyploid incidence and evolution. Annu. Rev. Genet. 34: 401-437. 
Rocha-Olivares, A., J.W. Fleeger \& D.W. Foltz. 2001. Decoupling of molecular and morphological evolution in deep lineages of a meiobenthic harpacticoid copepod. Mol. Biol. Evol. 18: 1088-1102.

Rohlf, F.J. 1990. Morphometrics. Annu. Rev. Ecol. Syst. 21: 299-316.

Rosset, S.D. 2008. New species of Odontophrynus Reinhardt and Lütken 1862 (Anura: Neobatrachia) from Brazil and Uruguay. J. Herpetol. 42: 134-144.

Rosset, S., D. Baldo, C. Lanzone \& N.G. Basso. 2006. Review of the geographic distribution of diploid and tetraploid populations of the Odontophrynus americanus species complex (Anura: Leptodactylidae). J. Herpetol. 40: 465-477.

Sáez, F.A. \& N. Brum-Zorrilla. 1959. Citogenética de anfibios anuros de América del Sur. Los cromosomas de Odontophrynus americanus y Ceratophrys ornata. An. Fac. Med. (Montevideo) 44: 414-423.

Salas, N.E. 2004. Las especies del género Odontophrynus de la provincia de Córdoba, Argentina. Tesis de Doctorado, Universidad Nacional de Río Cuarto, Córdoba, Argentina.

Savage, J.M. \& J.M. Cei. 1965. A review of the Leptodactylid frog genus Odontophrynus. Herpetologica 21: $178-195$.

Schäuble, C.S. 2004. Variation in body size and sexual dimorphism across geographical and environmental space in the frogs Limnodynastes tasmaniensis and $L$. peronii. Biol. J. Linn. Soc. 82: 39-56.

Shine, R. 1979. Sexual selection and sexual dimorphism in the Amphibia. Copeia 1979: 297-306.

Sinsch, U. \& H. Schneider. 1999. Taxonomic reassessment of Middle Eastern lake frogs: Morphological variation among populations considered as Rana ridibunda, R. bedriagae or R. levantina. J. Zool. Syst. Evol. Res. 37: 67-73.

Sinsch, U., A.W. Salas \& V. Canales. 1995. Reassessment of central Peruvian Telmatobiine (genera Batrachophrynus and Telmatobius). Morphometry and classification. Alytes 13: 14-44.

Taborsky, B., L. Guyer \& M. Taborsky. 2009. Size-assortative mating in the absence of mate choice. Anim. Behav. 77: 439-448.

Toledo, L.F., M.V. Garey, T.R.N. Costa, R. Lourenço-deMoraes, M.T. Hartmann \& C.F. B. Haddad. 2011. Alternative reproductive modes of Atlantic forest frogs. J. Ethol. Publicado online: 30 Diciembre 2011. DOI 10.1007/s10164-011-0322-9.

Tonini, J.F.R., I.S. Mendonça, A.B. Coutinho \& J.L. Gasparini. 2011. Anurans from Costa Bela, state of Espírito Santo, southeastern Brazil: inventory at an urban area and the re-discovery of Allobates in the state. Herpetol. Notes 4: 435-444.

Woolbright, L.L. 1983. Sexual selection and size dimorphism in anuran amphibians. Am. Nat. 121: 110-119. 
